\title{
Comparative Transcriptome Analysis Provides Insights into The Response of Ulva Compressa to The Fluctuating Salinity Conditions
}

\section{Qikun Xing}

Ocean University of China https://orcid.org/0000-0002-0617-9027

Guiqi Bi

Ocean University of China

Min Cao

Ocean University of China

\section{Arnaud Belcour}

Inria Centre de Recherche Rennes Bretagne Atlantique

\section{Méziane Aite}

Inria Centre de Recherche Rennes Bretagne Atlantique

Yunxiang Mao ( $\square$ yxmao@hntou.edu.cn )

Ocean University of China

\section{Research article}

Keywords: Ulva compressa, Hypo-salinity stress, Macroalgal blooms, Transcriptome, Long non-coding RNA

Posted Date: August 19th, 2020

DOI: https://doi.org/10.21203/rs.3.rs-47388/v1

License: @ (1) This work is licensed under a Creative Commons Attribution 4.0 International License. Read Full License

Version of Record: A version of this preprint was published at Journal of Phycology on June 2nd, 2021. See the published version at https://doi.org/10.1111/jpy.13167. 


\section{Abstract}

Background: Ulva compressa, known as the green tide forming species, was reported that can adapt to hypo-salinity conditions such as estuaries and brackish lakes. To understand the underlying molecular mechanisms of hypo-salinity stress tolerance, a genome-wide gene expression profiles in $U$. compressa was performed using digital gene expression profile (DGE).

Results: The RNA-seq data were analyzed based on the comparison of differently expressed genes involved in specific pathways under hypo-salinity and recovery conditions. Under the long-term hypo-salinity stress, the recovery of photosynthesis and energy metabolism could provide sufficient energy for the tolerance under long-term hypo-saline stress. Multiple strategies were performed to maintain the osmotic homeostasis. Additionally, several long non-coding RNA were detected as differently expressed genes during the stress, which could play important roles in the osmotolerance.

Conclusions: Our work will serve as an essential foundation for the understanding of the tolerance mechanism of $U$. compressa under the fluctuating salinity conditions.

\section{Background}

The excessive growth of green macroalgae have been reported in oceans around the world(1-3). Since 2007, massive green tide formed by Ulva species occurred in Yellow sea of China during the summer(4). Ulva spp. are able to tolerate various stress conditions such as temperature, light and salinity stress and grow rapidly when the condition is favorable to their growth(5). The green macroalgae blooming near the coastal area can be destructive to the local marine habitats and also cause major impacts on the economy of marine industries(6).

The green macroalgal species Ulva compressa, well known as green tide forming macroalgae, is widespread worldwide and it is dominant along coasts during spring and summer. Like other Ulva species, U. compressa can adapt to different stresses. Some of those have been widely studied such as desiccation and heavy metal stress(7-9). U. compressa are also frequently found in brackish environments such as estuaries and brackish lakes(10), where salinity were significantly varied because of freshwater inputs from rivers, rainfall and tidal period. In laboratory, $U$. compressa were tolerant to a wide range of salinities (5-50 psu) and maintained high growth rate at extreme salinity conditions (5psu and 50psu)(11). Therefore, $U$. compressa is a good model to explore the adaptation mechanism of macroalgae to fluctuated salinity.

As a pervasive abiotic factor, fluctuating salinity can cause ionic, salinity and oxidative stress, which have a strong effect on cellular function and organismal development in photosynthetic organisms. Under salinity stress, the hypo/hyper-osmolarity disturb the turgor pressure, ion distribution and organic solutes in the cell, generating reactive oxygen species (ROS), which otherwise cause oxidative destruction to cell (12-16). Long-term exposure to hyper/hypo salinity environments restrict cell division and may result in stunted growth (17). As the dominant vegetation in the intertidal and subtidal, macroalgae must be able to withstand changes in salinity (rain, evaporation). Recently, with the improvement of RNA sequencing approaches, transcriptomic analysis has started to be used in investigating the molecular response under the hyposalinity stress in algae. In the broadly halotolerant green alga, Picochlorum strain SENEW3, the main strategy towards hyposalinity stress is maintaining salinity equilibrium by suppressing the synthesis pathway of some osmolytes such as proline, trehalose and sorbitol(18). In brown alga Saccharina latissima, the low salinity stress induced antioxidant responses and repress the gene expression of photosynthesis(19). The inhibitory effect of hyposalinity stress on photosynthesis were also observed in another green alga Dunaliella salina (20). In Ulva species, however, only studies of biomedical and physiological response have been performed to reveal their response and adaptation to salinity stress. In Ulva fasciata, the long-term hypo- and hyper-salinity treatment reduced growth rate and caused oxidative damage to cells(21). The affection of carbon and nitrogen metabolism were also observed in Ulva pertusa during hypo- and hyper-salinity treatment(22). And the accumulation of free proline suggested that proline could be an important osmolyte in this species. Likewise, the dimethylsulfoniopropionate (DMSP) production was induced by hyper-salinity stress in Ulva fenestrata, indicating that DMSP could also be the osmolyte in U/va species(23). To better understand the mechanism of acclimation to fluctuated salinity, transcriptional regulation of related pathways still need to be explored.

In this work, digital gene expression (DGE) of 27 samples were performed in order to investigate the gene expression and regulation in response to hyposaline stress and recovery from stress in $U$. compressa. By comparing the RNA-seq data of hyposaline stress and recovery, we identified some key genes and pathways involved in responses to salinity stress in $U$. compressa. The findings of the present study will lay the foundation for elucidating the molecular mechanisms of salinity reduction and provide useful information for further study of $U$. compressa. 


\section{Method}

\subsection{Plant materials}

The algae were collected from the Ulva blooming area near estuaries of Jiangsu Province. Living material was brought back to laboratory and cleaned using sterilized seawater and a soft brush. Then those samples were identified using DNA barcoding. The thallus identified as $U$. compressa were cut into small piece to release spores. After the spores grew into small thallus, they were picked out as unialgal culture and kept in bubbling natural seawater with Provasoli's enrichment solution medium (PES) under $50 \mu \mathrm{mol}$ photons $\mathrm{m}^{-2} \mathrm{~s}^{-1}$ at $20 \pm 1^{\circ} \mathrm{C}$ and a $12: 12$ light: dark (L: D) photoperiod.

\subsection{Experimental design and sampling}

A global transcriptome for $U$. compressa was generated as a reference because of the limited genome information of this species. The material included samples from different treatments. After the treatment, the samples were frozen in liquid nitrogen and stored in $-80^{\circ} \mathrm{C}$.

For DGE analysis, the gametophytes were transferred from normal condition to hyposaline condition ( $5 \mathrm{psu}$ ) for three days. During this period, four time spots $(1 \mathrm{~h}, 6 \mathrm{~h}, 24 \mathrm{~h}, 3 \mathrm{~d})$ were chosen to harvest samples. For the salinity recovery, the algae were changed back to natural sea water (30 psu) after a three-day pre-incubation in low-salinity condition (5psu). The same time spots were used to harvest samples. Three independent replicates were used for each treatment. After every treatment, all samples were stored in $-80^{\circ} \mathrm{C}$ for RNA isolation.

\subsection{RNA isolation, sequencing and transcriptome assembly}

Total RNA was extracted by the Plant RNA Kit (Omega, U.S.) following the manufacturer's protocol. The quality of RNA was examined using Qubit 2.0 Fluorometer (Life Technologies, CA, USA) and the Bioanalyzer 2100 system (Agilent Technologies, CA, USA). After library preparation, the libraries were sequenced on an Illumina Hiseq XTen platform. Together, the library from the pooled RNA sample was sequenced with the 125-bp pair-end reads for de novo transcriptome analysis. The other twenty-seven libraries were sequenced with the 75-bp single-end reads for DGE analysis.

The quality of the Illumina reads was checked using FastQC. Reads were cleaned by removing adapters, low quality reads (Phred score $<33$ ) and short reads (<50 nucleotides) with Trimmomatic(24). De novo transcriptome assembly was performed using Trinity (v2.5.1) with all parameters set default(25). Then transcript abundance was estimated by RSEM(26) and transcripts with low expression level $($ TPM $<5)$ were removed. Transcriptome annotation was performed with a Blastx search against the NCBI-nr and the Swissprot database with an E-value cut-off of $10^{-5}$. The interproscan and GO annotation were performed on the Blast2GO (27). And KEGG pathways were assigned to the transcripts using the online KEGG Automatic Annotation Server, (http://www.genome.jp/kegg/kaas/). The singledirectional best hit (SBH) method was used for KEGG Orthology (KO) assignment.

\subsection{Metabolic network reconstruction}

Using the annotation from Blast2GO, especially the EC number, a genbank file was created with in-house Python scripts. This file was given as an input to Pathway Tools version 22.0(28). Using the gene annotations, Pahtway Tools predicted biochemical reaction and metabolic pathways. This information was stored in a Pathway Genome DataBase (PGDB). Using the PADMet toolbox version 2.6(29), the PGDB was converted into a file compatible with the so-called "PADMet format", which was used to create a wiki website (https://gemaureme.genouest.org/ucomgem/index.php/Main_Page). The website integrates both the prediction of Pathway Tools (genes, metabolites, reactions, pathways) and gene expression data.

\subsection{Differentially expressed genes identification and functional enrichment analysis}

Single-end reads were mapped to the reference transcriptome using Bowtie2 and expression of transcripts were calculated by RSEM(30). Then DESeq2 was used for the differential expression analysis by comparing control and treatment samples(31). A corrected P-value of 0.05 and |log2fold-changel > 1 were set as the threshold for significant differential expression.

GO and KEGG enrichment analysis of differentially expressed genes was performed using the TBtools software(32). GO terms and KEGG pathways with corrected P-values below 0.05 were considered significantly enriched by differentially expressed genes.

\subsection{Prediction of long non-coding RNAs and their targetgenes}

Page 3/18 
As described by Li et al (33), three criteria were used to identify long non-coding RNAs: 1) the transcripts that have no hits with known protein-coding genes on the same strand; 2 ) the transcripts have no coding potential according to the result of Coding Potential Calculator (CPC)(34), and Coding-Non-Coding Index (CNCI)(35);3) the transcripts have no hits with known protein domains in Pfam database(36). The transcripts complied with all of three criteria were considered as reliable IncRNAs. Differentially expressed (DE) IncRNAs were pair-wisely identified setting false discovery rate $<0.05$ and |log2fold-changel $>1$.

For construction of IncRNA-mRNA trans-regulation network, DE IncRNAs, together with DE genes, were subjected to the standard procedure of WGCNA(37). In the network, linked IncRNAs and genes were considered as co-expressed genes and potentially in transregulation.

\subsection{Quantitative real-time PCR (qRT-PCR) validation}

After RNA extraction, the first-strand cDNA synthesis was performed using First Stand cDNA Synthesis Kit (Roche). The qRT-PCR reactions were performed in 384-well plates in a LightCycler 480 (Roche Molecular Biochemicals, Mannheim, Germany) with LightCycler ${ }^{\circledR} 480$ SYBR Green I Master kit (Roche, Germany). The protocol was: $95^{\circ} \mathrm{C}$ for 5 min, followed by 55 cycles of $95^{\circ} \mathrm{C}$ for $10 \mathrm{~s}$, $60{ }^{\circ} \mathrm{C}$ for $10 \mathrm{~s}$, and $72{ }^{\circ} \mathrm{C}$ for $20 \mathrm{~s}$. Three biological replicates were performed for each treatment. Ubiquitin conjugating enzyme (UBC) and translation initiation factor 4A (elF4a). A 1:10 dilution series of the $U$. compressa cDNA was prepared and tested for each gene to determine the amplification efficiency, which was calculated by the LightCycle ${ }^{\circledR} 480$ gene scanning software (version 1.5 ). the raw data were divided by the $\mathrm{Cq}$ value of corresponding reference genes as a normalization step. And then the relative expression level of treated samples versus control samples was calculated by comparing the normalized $\mathrm{Cq}$ of treatment and control samples according to the formula: $2^{-\Delta \Delta C t}(\Delta \Delta C t=$ normalized Cq value of treated sample-normalized Cq value of control sample).

\section{Result}

\subsection{Global transcriptome assembly and annotation}

In order to obtain as many transcripts as possible, A mixed library of different abiotic stress conditions was sequenced and assembled (Additional file 1). A total of $2.17 \times 10^{8}$ filtered reads (without adaptor and low-quality bases) were obtained and the average GC content was $57 \%$. After assembly, the global transcriptome contained 19,079 transcripts with the length ranging from 201 to $9,987 \mathrm{bp}$. The mean length of transcripts was $1,409 \mathrm{bp}$. The annotation of global transcriptome was performed using different database: NCBI non-redundant protein sequences (Nr), Swiss-prot, Kyoto Encyclopedia of Genes and Genomes database (KEGG), Protein family (PFAM), and Clusters of Orthologous Groups of proteins (COG). In total, 10,045 $852.65 \%$ \unigenes were similar to proteins in at least one of these databases.

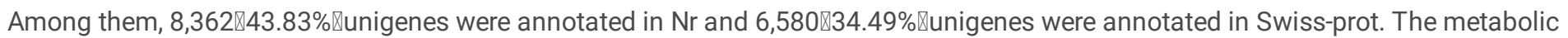
network reconstructed by Pathway Tools contained 1117 reactions, 1391 metabolites and 741 pathways. The wiki website containing those reactions, pathways and all the differential expressed genes involved in those pathways is available at: https://gemaureme.genouest.org/ucomgem/index.php/Main_Page. In this study, the wiki was used to explore the glycolysis (https://gemaureme.genouest.org/ucomgem/index.php/GLYCOLYSIS) and the TCA cycle (https://gemaureme.genouest.org/ucomgem/index.php/TCA) pathways.

\subsection{Analysis of differentially expressed gene}

To explore the gene expression pattern of $U$. compressa under hypo-salinity stress, a total of 27 RNA libraries generated from three replicates under the nine treatments were sequencing on a Nextseq500 platform. Raw data generated in each library ranged from 10.0 to 13.2 million reads. After removing low quality data, approximately 9.8-12.1 million clean reads remained, of which around $90 \%$ reads were mapped to the reference transcriptome. DESeq analysis were performed to identify the differentially expressed genes (DEGs) from read count data of various treatments. The thresholds of DEGs was set as the false discovery rate (FDR) $\leq 0.01$ and $\mid \log _{2}$ Ratio| $\geq 1$. The numbers and relationships of DEGs between different stress conditions were shown in Fig.1.

\subsection{DEGs related to Photosynthesis}

The hypo-salinity treatment caused a differential expression of 56 photosynthesis related genes. According to the heatmap (Fig.3, A), those genes were divided into two groups. The first group contained 22 genes which were significantly suppressed within 6 hours and up-regulated after 24 hours, coding for enzymes involved in light harvesting complex (e.g. Chlorophyll a-b binding protein of LHCll type I and protein CP29), photosystem II complex (e.g. Oxygen-evolving enhancer protein 1), Calvin cycle (e.g. glyceraldehyde 3-phosphate 
dehydrogenase, fructose-1,6-bisphosphatase and malate dehydrogenase) and photosynthetic electron transport chain (e.g. flavodiiron protein). The second group contains genes that were upregulated under both short term $(1 \mathrm{~h}, 6 \mathrm{~h})$ and long term $(24 \mathrm{~h}, 72 \mathrm{~h})$ of hyposalinity stress. Among those genes, 11 DEGs related to the photosystem maintenance or photosynthetic acclimation, such as HCF244 protein, MPH2 acclimation factor, YCF4 protein, HHL1 protein and VIPP protein.

When culture medium was change back to the natural seawater, the recovery of salinity caused a repression of 37 photosynthesis related genes. Except the genes suppressed under hypo-salinity stress, genes involved in photosystem I and photorespiration were also downregulated. Unlike the hypo-salinity condition, these genes were down-regulated through all three days. And the recovery condition induced 10 genes related to the photosystem maintenance or photosynthetic acclimation after 6 hours.

\subsection{DEGs related to glycolysis and TCA cycle}

After 1 hour treatment, the low salinity stress seemed to induce a downregulation of genes involved in glycolysis, such as Glucose-6phosphate isomerase, Phosphoglycerate kinase, 2,3-bisphosphoglycerate-independent phosphoglycerate mutase, Pyruvate kinase and Pyruvate dehydrogenase(Fig.4). At 24 hours, the expression of these genes increased back to normal. The transcripts of two enzymes in glycolysis were upregulated in very short time ( $1 \mathrm{~h}$ or $6 \mathrm{~h}$ ) after the hypo-salinity treatment. Similarly, the result clearly showed that $5 \mathrm{DEGs}$ related to TCA cycle have the same expression pattern with glycolysis related DEGs. However, the significantly downregulation of citrate synthase and isocitrate dehydrogenase was observed at $24 \mathrm{~h}$ of hypo-salinity stress.

In the salinity recovery process, most of DEGs associated with glycolysis and TCA cycle were significantly upregulated in very short time (1h or $6 \mathrm{~h}$ ) except Phosphoglycerate kinase and citrate synthase, indicating a quick activation of cellular respiration.

\subsection{Cellular transport and osmolytes metabolism}

Under the hypo-salinity stress, we detected 10 DEGs related to ion transporter such as sodium antiporter, chloride anion channel, nitrate transporter and calcium transporter(Table.1). One ABC transporters were repressed at $6 \mathrm{~h}$ and upregulated after $24 \mathrm{~h}$. Regarding the osmolytes metabolism, a DEG encoding trehalase was upregulated within $72 \mathrm{~h}$, which related to the trehalose degradation. In the proline synthesis pathway, the ornithine aminotransaminase transcript was downregulated within $72 \mathrm{~h}$. Similarly, gene coding for the choline dehydrogenase, which involved in the glycine betaine biosynthesis pathway, was downregulated after $72 \mathrm{~h}$.

During the salinity recovery process, 20 transporter genes were significantly differential expressed. Most of these genes were upregulated after $1 \mathrm{~h}$, including some and solute transporters. Unlike hypo-salinity stress, three ion channels and two ABC transporters were repressed during the recovery process. For the osmolytes metabolism, a DEG encoding 1-pyrroline-5-carboxylate synthetase in proline synthesis pathway was upregulated after $1 \mathrm{~h}$. Three choline dehydrogenase genes were also upregulated after $6 \mathrm{~h}$ of recovery.

\subsection{General stress responses}

During the hypo-salinity stress, a M-type thioredoxin gene and a dehydroascorbate reductase were induced after $24 \mathrm{~h}$ and $72 \mathrm{~h}$ separately(Table.2). And a DEG encoding glutathione reductase was upregulated after $1 \mathrm{~h}$ and kept high expression level throughout the whole stress process. Several DEGs coding cyclophilin-type peptidyl-prolyl cis-trans isomerase were upregulated within $6 \mathrm{~h}$ after the hyposalinity stress. Besides, two FKBP-type peptidyl-prolyl cis-trans isomerase and a Hsp70 chaperone were also upregulated after $1 \mathrm{~h}$ of stress treatment. A DEG coding ornithine decarboxylase was upregulated within $72 \mathrm{~h}$ of hypo-salinity stress, which could increase the biosynthesis of putrescine.

After $6 \mathrm{~h}$ of salinity recovery, four DEGs related to ROS scavenging were significantly upregulated such as glutathione reductase, thioredoxin and ascorbate peroxidase. Comparing to the hypo-salinity stress, more DEGs involved in the protein modification were upregulated such as NAC ribosome-associated chaperone and ZEF Hsp40-chaperone. In the polyamine synthesis pathway, six DEGs were upregulated, which related to the biosynthesis of putrescine, spermidine and thermospermine.

\subsection{Long non-coding RNAs prediction and IncRNA-mRNA interaction network}

In total, 2297 transcripts were identified as the potential long non-coding RNAs, among which 256 and 208 transcripts were significantly differential expressed under hypo-salinity stress and recovery process separately (Additional file 2). Since most IncRNAs regulated transtarget genes, a IncRNA-mRNA trans-regulated interaction network related to the hypo-salinity stress and recovery process(Fig.5). In this network, DEGs related to photosynthesis, TCA and chaperone were enriched and extracted. As shown in picture, there are 11 mRNA interacting with 27 IncRNA in the TCA sub-network. In the chaperone sub-network, mRNA and IncRNA had the same number of 22 . While

Page 5/18 
in the photosynthesis sub-network, 32 mRNA and 18 IncRNA were associated together and these IncRNA have more connectivity comparing to TCA and chaperone sub-network.

\subsection{Validation of RNA-Seq-based gene expression}

To validate the expression data obtained from RNA-seq, seven genes were randomly selected from differential expressed genes (Additional file 3). And qRT-PCR were performed to validate the expression level of each gene under the same stress conditions with RNAseq data. As shown in the figure, the qRT-PCR results of all genes have the same trend with the RNA-seq data(Additional file 3).

\section{Discussion}

The study present a profile of the global transcriptomic change during the hypo-salinity stress and salinity recovery treatment in $U$. compressa, which can tolerant a low-salinity environment. A whole-transcriptome expression analysis was processed under control and eight stress treatment conditions to reveal the molecular mechanisms of the hypo-salinity stress tolerance in $U$. compressa. The analysis of the DGE demonstrated that the responses of $U$. compressa under hypo-salinity stress were enhance the genes related to protein repair, salinity homeostasis, photosynthesis and ROS scavenging. And the biological processes during the salinity recovery mainly focus on intracellular osmolytes synthesis and DNA repair. Most of the stress-regulated genes are late-response gene, which contributed to the long-term tolerance of hypo-salinity stress in U. compressa.

\subsection{Multiple strategies of osmoregulation}

Under salinity stress, the hypo/hyper-salinity disturb the turgor pressure and cause the efflux/influx of inorganic ion and water, which has a strong effect on the regular biological processes. For example, in yeast, the exposure of cells to hypo-salinity shock results in a rapid inflow of water, which will cause the cell swelling and rupture(38). Generally, there are two basic responses to adjust the turgor pressure under salinity stress, ion transport and metabolic changes(39). When suffering from salinity stress, cells can rapidly restore their volume by activating ion channel on the membrane and transporting ion across the membrane(40). In present study, many genes encoding ion transporter protein were detected as up-regulated gene in the early stage of hypo-salinity stress, which indicate that active ion transportation was the main strategy of maintaining the turgor pressure in $U$. compressa under short-term hypo-salinity stress.

Organic osmolytes are soluble and compatible compounds within cells of almost all organisms, of which the amount changes in response to salinity stress. Previous studies have identified various osmolytes in different species. Proline is widely believed to be characteristic osmolytes for most Chlorophyceae(41). When expose to hypo-salinity stress, algae tended to decrease the biosynthesis of osmolytes to reduce the turgor pressure. In our study, gene encoding ornithine aminotransaminase were significantly downregulated after $72 \mathrm{~h}$ of hypo-salinity treatment. Ornithine aminotransaminase (OAT) is a pyridoxal-5'-phosphate-dependent enzyme that has been proposed to be involved in proline (Pro) and arginine (Arg) metabolism. Overexpression of OAT gene in rice significantly increased proline accumulation and enhanced the osmotic stress resistance(42). Hence, we infer that the repression of gene coding for $O A T$ might ultimately lead to the decrease of proline during the hypo-salinity stress to reduce the turgor pressure. Another key enzyme in proline biosynthesis pathway, $\Delta^{1}$-pyrroline-5-carboxylate synthetase ( $\left.P 5 C S\right)$, convert glutamate to $\Delta^{1}$-pyrroline-5-carboxylate. In transgenic potato, the overexpression of P5CS can also result in the Pro accumulation(43). One gene encoding P5CS was significantly upregulated under the salinity recovery process, which could cause the accumulation of proline and adjust the turgor pressure. In the diatom Thalassiosira pseudonana, the biosynthesis of glycine betaine via choline dehydrogenase was induced under hypersaline conditions(44). Here we found one choline dehydrogenase gene was significantly downregulated under the hypo-salinity stress and three choline dehydrogenase genes showed significantly upregulated in the recovery process, which means the glycine betaine could also participated in the osmoregulation process as osmolytes in $U$. compressa. As a natural non-reducing sugar, trehalose is believed to play osmoprotectant role and carbon source in many species(45). In our study, one trehalase genes was upregulated during the hypo-salinity stress, which could degrade trehalose to adjust the turgor pressure.

\subsection{Energy metabolism}

When algae exposed to hypo-salinity stress, ROS production was induced in cells, resulting in the damage to PSII complex and the decrease in overall activity of photosynthesis(20). In higher plants, hyper-salinity stress inhibited the carbon fixation by curtailing the activity of ribulose-1,5-bisphosphate carboxylase, which catalyzed the first step of photosynthetic $\mathrm{CO}_{2}$ assimilation(46). Similarly, in Dunaliella viridis, the activity of Rubisco and the expression of Rubisco large subunit $(r b c \mathrm{~L})$ were inhibited by the hypo-salinity stress $(47)$. 
In the present study, 10 genes involved in C3 pathway up-regulated after 24h hypo-salinity stress treatment, indicating that U.compressa restored the activity of carbon fixation under long-term hypo-salinity stress.

The electron transport chain (ETC) in chloroplast transfers electrons from photosystem reaction center to Calvin cycle via reduction and oxidation of a series of complexes. After the inhibition of $\mathrm{CO}_{2}$ fixation, most of excitation energy couldn't be utilized by Calvin cycle, resulting in the overreduction of the electron transport chain and enhanced generation of ROS(48). A total of six genes encoding electron transport chain protein were showed significantly upregulated along with the genes in C3 pathway. It is supposed that the repair of overreduced electron transport chain was performed to enhance the tolerance of hypo-salinity stress.

The reaction center of PSII was very sensitive to the environmental stress and easily damaged by the light-dependent destruction. Then the ROS produced in chloroplast inhibited the repair of PSIl by suppressing the synthesis of photosynthesis-related protein, which prolonged the period of photoinhibition(49). In Arabidopsis thaliana, most of photosynthesis-related genes were suppressed under hypersalinity and drought stress(50). In red algae Gracilaria changii, repression of the genes encoding light-harvesting proteins under hyposaline stress(51). The down-regulation of photosynthesis related genes was also observed in brown algae Ectocarpus siliculosus under hyposaline(52). In this study, short-term hyposaline stress repressed genes coding light-harvesting proteins and PSII oxygenevolving enhancer protein. However, these genes were up-regulated under long-term hypo-salinity stress, together with genes coding components of photosynthesis reaction center. The upregulation of these genes supposed that the repair of photosystem was activated by enhancing the expression of photosynthesis-related genes. Moreover, many genes encoding proteins related to the photosystem assembly and maintenance, such as YCF4 protein, HHL1 protein and TerC protein, were also detected as upregulated DEGs under both short-term and long-term hypo-salinity stress. In green algae Chlamydomonas reinhardtii and land plants, these proteins are required for stable accumulation of the PSI and PSII complex(53-55). The generation of photosynthesis-related protein maintained the high rate of light reaction in $U$. compressa, providing energy and metabolites needed for the tolerance of hypo-salinity stress. During the salinity recovery process, the repression of genes coding components of photosynthesis reaction center was observed at $1 \mathrm{~h}$ and $6 \mathrm{~h}$. Comparing to the hyposaline stress, more genes related to photosystem maintenance and photo protection were significantly upregulated during the whole process. The result might indicate that the salinity recovery didn't cause strong damage on the photosynthesis reaction center.

Under the hyposaline condition, algae need sufficient supply of energy to adapt to the stress, including osmolytes accumulation and protein synthesis(56). In our study, many glycolysis genes were repressed at $1 \mathrm{~h}$ of hypo-salinity stress. After that, the expression level of these genes rose back to normal after $24 \mathrm{~h}$. Transcripts encoding fructose-bisphosphate aldolase and dihydrolipoyl transacetylase were significantly up-regulated during the hyposaline stress. Fructose-bisphosphate aldolase catalyzes the reverse cleavage of fructose-1,6bisphosphate into dihydroxyacetone phosphate (DHAP) and 3-phosphate glyceraldehyde (G-3-P). In land plant Brassica napus, the overexpression of fructose-bisphosphate aldolase gene could increase the survival ability under salt stress conditions. Unlike the glycolysis pathway, two rate-limiting enzymes, citrate synthase and isocitrate dehydrogenases, were repressed after $24 \mathrm{~h}$ of hypo-salinity stress, indicating the low activity of TCA pathway. In summary, the recovery of glycolysis activity could provide energy for the stress tolerance. However, in the recovery process, genes involved in glycolysis and TCA pathway were upregulated, which will provide sufficient supply of energy for the cell recovery.

\subsection{Cellular protection processes}

ROS are frequently accumulated when plants expose to abiotic stress, causing oxidative damage to cellular components(57). To avoid the oxidative damage, plants always activate a set of anti-oxidation mechanism under abiotic stress. Genes encoding glutathione reductase and thioredoxin were detected during the whole hypo-salinity stress and recovery period. Glutathione reductase catalyzes the reduction of glutathione disulfide to the sulfhydryl form glutathione, which is a critical molecule in resisting oxidative stress and maintaining the reducing environment of the cell. In the red algae Gracilaria corticata, the activity of glutathione reductase increased under both hypo-salinity stress and hyper-salinity(58). The up-regulation of gene encoding glutathione reductase was also observed in Ulva faciata under hypersalinity (59). Thioredoxin is a small $(12 \mathrm{kDa})$ thiol-active protein important for maintaining intracellular redox status. Gene coding thioredoxin in U. compressa was induce to protect cell under oxidative stress(60). The upregulation of these two genes indicates the oxidative stress was induced in both hypo-salinity stress and recovery process. Additionally, we also found an ascorbate peroxidase gene only induced by the recovery process, indicating that ascorbate peroxidase could play an important role in ROS scavenging in recovery process.

The protection of cellular components plays a crucial role in tolerating the abiotic stress by maintaining cellular homeostasis. It is wellknown that heat-shock proteins (Hsps) contribute greatly to cell protection by responding for folding, assembly, translocation and degradation in many normal cellular processes, stabilizing proteins and membranes, and assisting in protein refolding under stress

Page $7 / 18$ 
conditions $(61,62)$. Based on subunit sizes, five major families of Hsps are conservatively recognized: the Hsp70 (DnaK) family; the chaperonins (GroEL and Hsp60); the Hsp90 family; the Hsp100 (Clp) family; and the small Hsp (sHsp) family(63). In our study, two Hsp70 genes were detected upregulated in recovery process. Hsp 70 constitute a set of cellular machines that assist the folding of newly translated proteins, transport of precursor proteins across membranes, scavenging the damaged protein and repair of misfolded proteins $(64,65)$. Previous studies showed that the overexpression of Hsp 70 genes result in enhanced tolerance to various abiotic stress in higher plants(66-69). The upregulation of Hsp70 genes during the recovery of salinity indicates that these genes could play an important role in cellular protein homeostasis while recovering from hypo-salinity stress. Moreover, one Hsp40 was detected specifically upregulated in recovery process. Hsp40 protein has been proved that it has the ability to regulate the formation of Hsp70 complex(70). The upregulation of Hsp40 gene could enhance the function of Hsp70 complex under recovery process. Besides, several FKBP-type and cyclophilin-type peptidyl-prolyl cis-trans isomerase showed upregulated under hypo-salinity stress. In Pyropia yezoensis, it has been proved that the purified protein of cyclophilin-type peptidyl-prolyl cis-trans isomerase was able to act against oxidative stress(71). And the FKBP-type peptidyl-prolyl cis-trans isomerase from chlorophycean microalga, Scenedesmus sp., can also enhance the tolerance of salinity stress(72).

Polyamines are involved in many fundamental cellular processes. In land plants, common polyamines are putrescine, spermidine and spermine. Polyamines can activate the signaling pathway related to the stress response and enhance the tolerance of stress(73). The biosynthesis pathways of polyamine are different between different plant or algae species(74). In our study, only one gene involved in the putrescine synthesis was significantly upregulated in hypo-salinity stress. However, more polyamine biosynthesis pathways, such as spermidine and thermospermine, were activated in the recovery process. Thermospermine is an isomer of spermine and also participate in the stress response of land plant and algae such as diatom Thalassiosira pseudonana and Arabidopsis thaliana(75). The induction of these genes indicates that polyamines might be essential in the salinity recovery process of $U$. compressa.

\subsection{Stress-related IncRNAs and their regulation network}

As a class of endogenous nonprotein-encoding transcripts, IncRNAs have complex and precise regulatory functions in gene expression of different development stage and stress conditions $(76,77)$. Recently, many researches have been done on stress-related IncRNA in land plants. Deng et al. found 44 differentially expressed IncRNA under salt stress in Gossypium hirsutum(78). In Manihot esculenta, 318 IncRNAs were identified responsive to cold and/or drought stress(79). Wang et al. found that IncRNA have various expression pattern in different tissue of Medicago truncatula under osmotic stress(80). However, few works have been done on the IncRNA in algae. LncRNAs were identified and reported in two brown algae (Ectocarpus siliculosus and Saccharina japonica) and one green algae (Chlamydomonas reinhardtii) (81, 82). The function and expression of algal IncRNA are mostly unknown. In our study, 256 and 208 transcripts were detected as differential expressed IncRNAs in hypo-salinity stress and recovery process. The IncRNA-mRNA co-expression network also indicates IncRNAs that might be involved in the regulation of photosynthesis, TCA and protein homeostasis, which play important roles in hypo-salinity stress and recovery process.

\section{Conclusions}

The combination of de novo transcriptome sequencing and DGE analysis based on the NGS technology is a powerful method for uncovering the molecular mechanism of the response to the salinity stress in $U$. compressa. In this study, we sketched the contours of the transcriptional regulation in $U$. compressa under hypo-salinity stress and recovery. The maintaining of photosystem and quick recovery of carbon metabolism provide sufficient energy for other accumulation process. With sufficient energy support, U. compressa use multiple osmoregulation strategies, such as inorganic ion transport, organic osmolytes transport and regulation of metabolism under hypo-salinity stress. Besides, active expression of heat shock protein genes and ROS scavenging genes were also considered as the reason of the tolerance to hypo-salinity stress in U. compressa. Additionally, many IncRNAs were detected in our study, which could play an important role in the osmotolerance. The transcriptome data generated in the present study can serve as an important resource for understanding tolerance mechanisms of $U$. compressa as it thrives in continually changeable environment.

\section{Declarations}

\section{Ethics approval and consent to participate}

Not applicable.

\section{Consent for publication}


Not applicable.

\section{Data availability statement}

The Illumina sequence reads generated during the present study are available in the NCBI SRA database under the BioProject ID: PRJNA643404.

accession number

\section{Competing interests}

The authors declare that they have no competing interests

\section{Funding information}

This work was financially supported by the Marine S\&T Fund of Shandong Province for Pilot National Laboratory for Marine Science and Technology (Qingdao) (No. 2018SDKJ0302-5), the Fundamental Research Funds for the Central Universities (201762016) \the Project of National Infrastructure of Fishery Germplasm Resources (2018DKA30470) and the MOA Modern Agricultural Talents Support Project. These funding bodies had no role in the study design, analysis, decision to publish or preparation of the manuscript.

\section{Authors' contribution}

QX and YM conceived the project. QX performed RNA-seq data analysis with help from GB. QX performed RNA extraction and sequencing library construction with help from MC. AB and MA performed the genome-scale metabolic network reconstruction. QX wrote the manuscript with input and edits from all authors.

\section{Acknowledgements}

We would like to thank Anne Siegel and Jeanne Got from Dyliss team for uploading and maintaining the metabolic network website page. This work has also benefited from the computational resources of the bioinformatic platform in College of Marine Life Sciences, Ocean University of China and ABiMS bioinformatics platform (FR 2424, CNRS-Sorbonne Université, Roscoff).

\section{References}

1. Hiraoka M, Ohno M, Kawaguchi S, Yoshida G. Crossing test among floating Ulva thalli forminggreen tide'in Japan. Hydrobiologia. 2004;512(1-3):239-45.

2. Morand P, Merceron M. Macroalgal population and sustainability. Journal of coastal research. 2005:1009-20.

3. Merceron M, Antoine V, Auby I, Morand P. In situ growth potential of the subtidal part of green tide forming Ulva spp. stocks. Science of the Total Environment. 2007;384(1-3):293-305.

4. Liu D, Keesing JK, Xing Q, Shi P. World's largest macroalgal bloom caused by expansion of seaweed aquaculture in China. Marine Pollution Bulletin. 2009;58(6):888-95.

5. Taylor R, Fletcher R, Raven J. Preliminary studies on the growth of selected 'green tide'algae in laboratory culture: effects of irradiance, temperature, salinity and nutrients on growth rate. Botanica Marina. 2001;44(4):327-36.

6. Ansell A, Gibson R, Barnes M, Press U. Ecological impact of green macroalgal blooms. Oceanography and Marine Biology: an annual review. 1998;36:97-125.

7. Holzinger A, Herburger K, Kaplan F, Lewis LA. Desiccation tolerance in the chlorophyte green alga Ulva compressa: does cell wall architecture contribute to ecological success? Planta. 2015;242(2):477-92.

8. González A, de Los Ángeles Cabrera M, Henríquez MJ, Contreras RA, Morales B, Moenne A. Cross talk among calcium, hydrogen peroxide, and nitric oxide and activation of gene expression involving calmodulins and calcium-dependent protein kinases in Ulva compressa exposed to copper excess. Plant Physiology. 2012;158(3):1451-62.

9. Navarrete A, González A, Gómez M, Contreras RA, Díaz P, Lobos G, et al. Copper excess detoxification is mediated by a coordinated and complementary induction of glutathione, phytochelatins and metallothioneins in the green seaweed Ulva compressa. Plant physiology and biochemistry. 2019;135:423-31. 
10. Ogawa T, Ohki K, Kamiya M. Differences of spatial distribution and seasonal succession among Ulva species (Ulvophyceae) across salinity gradients. Phycologia. 2013;52(6):637-51.

11. Wu H, Shin SK, Jang S, Yarish C, Kim JK, Wu H, et al. Growth and nutrient bioextraction of Gracilaria chorda, G. vermiculophylla, Ulva prolifera, and U. compressa under hypo-and hyper-osmotic conditions. Algae. 2018;33(4):329-40.

12. Collén J, Davison I. Stress tolerance and reactive oxygen metabolism in the intertidal red seaweeds Mastocarpus stellatus and Chondrus crispus. Plant, Cell \& Environment. 1999;22(9):1143-51.

13. Liu F, Pang SJ. Stress tolerance and antioxidant enzymatic activities in the metabolisms of the reactive oxygen species in two intertidal red algae Grateloupia turuturu and Palmaria palmata. Journal of Experimental Marine Biology and Ecology. 2010;382(2):82-7.

14. Choo K-s, Snoeijs P, Pedersén M. Oxidative stress tolerance in the filamentous green algae Cladophora glomerata and Enteromorpha ahlneriana. Journal of Experimental Marine Biology and Ecology. 2004;298(1):111-23.

15. Dring MJ. Stress resistance and disease resistance in seaweeds: the role of reactive oxygen metabolism. Advances in botanical research. 2005;43:175-207.

16. Wu T-M, Lee T-M. Regulation of activity and gene expression of antioxidant enzymes in Ulva fasciata Delile (Ulvales, Chlorophyta) in response to excess copper. Phycologia. 2008;47(4):346-60.

17. Kumar M, Kumari P, Gupta V, Reddy C, Jha B. Biochemical responses of red alga Gracilaria corticata (Gracilariales, Rhodophyta) to salinity induced oxidative stress. Journal of Experimental Marine Biology and Ecology. 2010;391(1):27-34.

18. Foflonker F, Ananyev G, Qiu H, Morrison A, Palenik B, Dismukes GC, et al. The unexpected extremophile: tolerance to fluctuating salinity in the green alga Picochlorum. Algal research. 2016;16:465-72.

19. Li H, Monteiro C, Heinrich S, Bartsch I, Valentin K, Harms L, et al. Responses of the kelp Saccharina latissima (Phaeophyceae) to the warming Arctic: from physiology to transcriptomics. Physiologia plantarum. 2019.

20. Liu W, Ming Y, Li P, Huang Z. Inhibitory effects of hypo-osmotic stress on extracellular carbonic anhydrase and photosynthetic efficiency of green alga Dunaliella salina possibly through reactive oxygen species formation. Plant Physiology and Biochemistry. 2012;54:43-8.

21. Lu I-F, Sung M-S, Lee T-M. Salinity stress and hydrogen peroxide regulation of antioxidant defense system in Ulva fasciata. Marine Biology. 2006;150(1):1-15.

22. Kakinuma M, Coury D, Kuno Y, Itoh S, Kozawa Y, Inagaki E, et al. Physiological and biochemical responses to thermal and salinity stresses in a sterile mutant of Ulva pertusa (Ulvales, Chlorophyta). Marine Biology. 2006;149(1):97-106.

23. Van Alstyne KL, Pelletreau KN, Rosario K. The effects of salinity on dimethylsulfoniopropionate production in the green alga Ulva fenestrata Postels et Ruprecht (Chlorophyta). Botanica Marina. 2003;46(4):350-6.

24. Bolger AM, Lohse M, Usadel B. Trimmomatic: a flexible trimmer for Illumina sequence data. Bioinformatics. 2014;30(15):2114-20.

25. Grabherr MG, Haas BJ, Yassour M, Levin JZ, Thompson DA, Amit I, et al. Trinity: reconstructing a full-length transcriptome without a genome from RNA-Seq data. Nature biotechnology. 2011;29(7):644.

26. Li B, Dewey CN. RSEM: accurate transcript quantification from RNA-Seq data with or without a reference genome. BMC bioinformatics. 2011;12(1):323.

27. Conesa A, Götz S, García-Gómez JM, Terol J, Talón M, Robles M. Blast2GO: a universal tool for annotation, visualization and analysis in functional genomics research. Bioinformatics. 2005;21(18):3674-6.

28. Karp PD, Paley S, Romero P. The pathway tools software. Bioinformatics. 2002;18(suppl_1):S225-S32.

29. Aite M, Chevallier M, Frioux C, Trottier C, Got J, Cortés MP, et al. Traceability, reproducibility and wiki-exploration for "à-la-carte" reconstructions of genome-scale metabolic models. PLoS computational biology. 2018;14(5):e1006146.

30. Langmead B, Salzberg SL. Fast gapped-read alignment with Bowtie 2. Nature methods. 2012;9(4):357.

31. Love MI, Huber W, Anders S. Moderated estimation of fold change and dispersion for RNA-seq data with DESeq2. Genome biology. 2014;15(12):550.

32. Chen $\mathrm{C}$, Chen $\mathrm{H}, \mathrm{He} \mathrm{Y,} \mathrm{Xia} \mathrm{R.} \mathrm{TBtools,} \mathrm{a} \mathrm{toolkit} \mathrm{for} \mathrm{biologists} \mathrm{integrating} \mathrm{various} \mathrm{biological} \mathrm{data} \mathrm{handling} \mathrm{tools} \mathrm{with} \mathrm{a} \mathrm{user-friendly}$ interface. BioRxiv. 2018:289660.

33. Li C-Y, Li X, Liu Z, Ni W, Zhang X, Hazi W, et al. Identification and characterization of long non-coding RNA in prenatal and postnatal skeletal muscle of sheep. Genomics. 2019;111(2):133-41.

Page 10/18 
34. Kang Y-J, Yang D-C, Kong L, Hou M, Meng Y-Q, Wei L, et al. CPC2: a fast and accurate coding potential calculator based on sequence intrinsic features. Nucleic acids research. 2017;45(W1):W12-W6.

35. Sun L, Luo H, Bu D, Zhao G, Yu K, Zhang C, et al. Utilizing sequence intrinsic composition to classify protein-coding and long noncoding transcripts. Nucleic acids research. 2013;41(17):e166-e.

36. Bateman A, Coin L, Durbin R, Finn RD, Hollich V, Griffiths-Jones S, et al. The Pfam protein families database. Nucleic acids research. 2004;32(suppl_1):D138-D41.

37. Langfelder P, Horvath S. WGCNA: an R package for weighted correlation network analysis. BMC bioinformatics. 2008;9(1):559.

38. Hohmann S. Shaping up: the response of yeast to osmotic stress. Yeast stress responses. 1997:101-46.

39. Xiong L, Zhu JK. Molecular and genetic aspects of plant responses to osmotic stress. Plant, Cell \& Environment. 2002;25(2):131-9.

40. Burg MB, Ferraris JD. Intracellular organic osmolytes: function and regulation. Journal of Biological Chemistry. 2008;283(12):730913.

41. Oren A. Diversity of organic osmotic compounds and osmotic adaptation in cyanobacteria and algae. Algae and Cyanobacteria in Extreme Environments: Springer; 2007. p. 639-55.

42. You J, Hu H, Xiong L. An ornithine $\delta$-aminotransferase gene OsOAT confers drought and oxidative stress tolerance in rice. Plant Science. 2012;197:59-69.

43. Hmida-Sayari A, Gargouri-Bouzid R, Bidani A, Jaoua L, Savouré A, Jaoua S. Overexpression of $\Delta 1$-pyrroline-5-carboxylate synthetase increases proline production and confers salt tolerance in transgenic potato plants. Plant Science. 2005;169(4):746-52.

44. Kageyama H, Tanaka Y, Takabe T. Biosynthetic pathways of glycinebetaine in Thalassiosira pseudonana; functional characterization of enzyme catalyzing three-step methylation of glycine. Plant Physiology and Biochemistry. 2018;127:248-55.

45. Kosar F, Akram NA, Sadiq M, Al-Qurainy F, Ashraf M. Trehalose: a key organic osmolyte effectively involved in plant abiotic stress tolerance. Journal of Plant Growth Regulation. 2019;38(2):606-18.

46. Sivakumar P, Sharmila P, Saradhi PP. Proline alleviates salt-stress-induced enhancement in ribulose-1, 5-bisphosphate oxygenase activity. Biochemical and biophysical research communications. 2000;279(2):512-5.

47. Wang D, Wang W, Xu N, Sun X. Changes in growth, carbon and nitrogen enzyme activity and mRNA accumulation in the halophilic microalga Dunaliella viridis in response to NaCl stress. Journal of Ocean University of China. 2016;15(6):1094-100.

48. Lawlor DW, Cornic G. Photosynthetic carbon assimilation and associated metabolism in relation to water deficits in higher plants. Plant, cell \& environment. 2002;25(2):275-94.

49. Murata N, Takahashi S, Nishiyama Y, Allakhverdiev SI. Photoinhibition of photosystem II under environmental stress. Biochimica et Biophysica Acta (BBA)-Bioenergetics. 2007;1767(6):414-21.

50. Kilian J, Whitehead D, Horak J, Wanke D, Weinl S, Batistic O, et al. The AtGenExpress global stress expression data set: protocols, evaluation and model data analysis of UV-B light, drought and cold stress responses. The Plant Journal. 2007;50(2):347-63.

51. Teo SS, Ho CL, Teoh S, Rahim RA, Phang SM. TRANSCRIPTOMIC ANALYSIS OF GRACILARIA CHANGII (RHODOPHYTA) IN RESPONSE TO HYPER-AND HYPOOSMOTIC STRESSES 1. Journal of phycology. 2009;45(5):1093-9.

52. Dittami SM, Scornet D, Petit J-L, Ségurens B, Da Silva C, Corre E, et al. Global expression analysis of the brown alga Ectocarpus siliculosus (Phaeophyceae) reveals large-scale reprogramming of the transcriptome in response to abiotic stress. Genome biology. 2009;10(6):R66.

53. Boudreau E, Takahashi Y, Lemieux C, Turmel M, Rochaix JD. The chloroplast ycf3 and ycf4 open reading frames of Chlamydomonas reinhardtii are required for the accumulation of the photosystem I complex. The EMBO journal. 1997;16(20):6095-104.

54. Jin H, Liu B, Luo L, Feng D, Wang P, Liu J, et al. HYPERSENSITIVE TO HIGH LIGHT1 interacts with LOW QUANTUM YIELD OF PHOTOSYSTEM II1 and functions in protection of photosystem II from photodamage in Arabidopsis. The Plant Cell. 2014;26(3):1213-29.

55. Plöchinger M, Schwenkert S, von Sydow L, Schröder WP, Meurer J. Functional update of the auxiliary proteins PsbW, PsbY, HCF136, PsbN, TerC and ALB3 in maintenance and assembly of PSII. Frontiers in plant science. 2016;7:423.

56. Dittami SM, Heesch S, Olsen JL, Collén J. Transitions between marine and freshwater environments provide new clues about the origins of multicellular plants and algae. Journal of phycology. 2017;53(4):731-45.

57. Hernandez M, Fernandez-Garcia N, Diaz-Vivancos P, Olmos E. A different role for hydrogen peroxide and the antioxidative system under short and long salt stress in Brassica oleracea roots. Journal of experimental botany. 2009;61(2):521-35. 
58. Kumar M, Kumari P, Gupta V, Reddy C, Jha B. Biochemical responses of red alga Gracilaria corticata (Gracilariales, Rhodophyta) to salinity induced oxidative stress. Journal of Experimental Marine Biology and Ecology. 2010;391(1-2):27-34.

59. Sung M-S, Hsu Y-T, Hsu Y-T, Wu T-M, Lee T-M. Hypersalinity and hydrogen peroxide upregulation of gene expression of antioxidant enzymes in Ulva fasciata against oxidative stress. Marine biotechnology. 2009;11(2):199.

60. Contreras-Porcia L, Dennett G, González A, Vergara E, Medina C, Correa JA, et al. Identification of copper-induced genes in the marine alga Ulva compressa (Chlorophyta). Marine biotechnology. 2011;13(3):544-56.

61. Feder ME, Hofmann GE. Heat-shock proteins, molecular chaperones, and the stress response: evolutionary and ecological physiology. Annual review of physiology. 1999;61(1):243-82.

62. Nelson RJ, Ziegelhoffer T, Nicolet C, Werner-Washburne M, Craig EA. The translation machinery and 70 kd heat shock protein cooperate in protein synthesis. Cell. 1992;71(1):97-105.

63. Wang W, Vinocur B, Shoseyov O, Altman A. Role of plant heat-shock proteins and molecular chaperones in the abiotic stress response. Trends in plant science. 2004;9(5):244-52.

64. Hartl FU. Molecular chaperones in cellular protein folding. Nature. 1996;381(6583):571.

65. Frydman J. Folding of newly translated proteins in vivo: the role of molecular chaperones. Annual review of biochemistry. 2001;70(1):603-47.

66. Alvim FC, Carolino SM, Cascardo JC, Nunes CC, Martinez CA, Otoni WC, et al. Enhanced accumulation of BiP in transgenic plants confers tolerance to water stress. Plant Physiology. 2001;126(3):1042-54.

67. Sung DY, Guy CL. Physiological and molecular assessment of altered expression of Hsc70-1 in Arabidopsis. Evidence for pleiotropic consequences. Plant Physiology. 2003;132(2):979-87.

68. Sugino M, Hibino T, Tanaka Y, Nii N, Takabe T. Overexpression of DnaK from a halotolerant cyanobacterium Aphanothece halophytica acquires resistance to salt stress in transgenic tobacco plants. Plant Science. 1999;146(2):81-8.

69. Leborgne-Castel N, Jelitto-Van Dooren EP, Crofts AJ, Denecke J. Overexpression of BiP in tobacco alleviates endoplasmic reticulum stress. The Plant Cell. 1999;11(3):459-69.

70. Fan C-Y, Lee S, Cyr DM. Mechanisms for regulation of Hsp70 function by Hsp40. Cell stress \& chaperones. 2003;8(4):309.

71. Kim EY, Choi YH, Choi CG, Nam TJ. Effects of the cyclophilin-type peptidylprolyl cis-trans isomerase from Pyropia yezoensis against hydrogen peroxide-induced oxidative stress in HepG2 cells. Molecular medicine reports. 2017;15(6):4132-8.

72. Subin C, Pradeep M, Vijayan K. FKBP-type peptidyl-prolyl cis-trans isomerase from thermophilic microalga, Scenedesmus sp.: molecular characterisation and demonstration of acquired salinity and thermotolerance in E. coli by recombinant expression. Journal of Applied Phycology. 2016;28(6):3307-15.

73. Sagor G, Takahashi H, Niitsu M, Takahashi Y, Berberich T, Kusano T. Exogenous thermospermine has an activity to induce a subset of the defense genes and restrict cucumber mosaic virus multiplication in Arabidopsis thaliana. Plant cell reports. 2012;31(7):122732.

74. Fuell C, Elliott KA, Hanfrey CC, Franceschetti M, Michael AJ. Polyamine biosynthetic diversity in plants and algae. Plant Physiology and Biochemistry. 2010;48(7):513-20.

75. Knott JM, Römer P, Sumper M. Putative spermine synthases from Thalassiosira pseudonana and Arabidopsis thaliana synthesize thermospermine rather than spermine. FEBS letters. 2007;581(16):3081-6.

76. Liu X, Hao L, Li D, Zhu L, Hu S. Long non-coding RNAs and their biological roles in plants. Genomics, proteomics \& bioinformatics. 2015;13(3):137-47.

77. Zhang Y-C, Chen Y-Q. Long noncoding RNAs: new regulators in plant development. Biochemical and biophysical research communications. 2013;436(2):111-4.

78. Deng F, Zhang X, Wang W, Yuan R, Shen F. Identification of Gossypium hirsutum long non-coding RNAs (IncRNAs) under salt stress. BMC plant biology. 2018;18(1):23.

79. Li S, Yu X, Lei N, Cheng Z, Zhao P, He Y, et al. Genome-wide identification and functional prediction of cold and/or droughtresponsive IncRNAs in cassava. Scientific reports. 2017;7:45981.

80. Wang T-Z, Liu M, Zhao M-G, Chen R, Zhang W-H. Identification and characterization of long non-coding RNAs involved in osmotic and salt stress in Medicago truncatula using genome-wide high-throughput sequencing. BMC plant biology. 2015;15(1):131.

81. Li H, Wang Y, Chen M, Xiao P, Hu C, Zeng Z, et al. Genome-wide long non-coding RNA screening, identification and characterization in a model microorganism Chlamydomonas reinhardtii. Scientific reports. 2016;6(1):1-15.

Page 12/18 
82. Cormier A, Avia K, Sterck L, Derrien T, Wucher V, Andres G, et al. Re-annotation, improved large-scale assembly and establishment of a catalogue of noncoding loci for the genome of the model brown alga Ectocarpus. New Phytologist. 2017;214(1):219-32.

\section{Tables}

Table. $1 \log _{2}$ foldchange value of genes related to osmoregulation under different conditions comparing to control

\begin{tabular}{|c|c|c|c|c|c|c|c|c|c|}
\hline \multirow[t]{2}{*}{ ID } & \multirow[t]{2}{*}{ Annotation } & \multicolumn{4}{|c|}{ Hypo-salinity } & \multicolumn{4}{|c|}{ Recovery } \\
\hline & & $1 \mathrm{~h}$ & $6 h$ & $24 \mathrm{~h}$ & $72 \mathrm{~h}$ & $1 \mathrm{~h}$ & $6 \mathrm{~h}$ & $24 \mathrm{~h}$ & $72 \mathrm{~h}$ \\
\hline TRINITY_DN19793_c0_g2_i3 & trehalase & 1.76 & 1.42 & 0.94 & 1.20 & - & - & - & - \\
\hline TRINITY_DN20824_c0_g1_i1 & $\begin{array}{l}\text { ornithine } \\
\text { transaminase }\end{array}$ & -2.43 & -1.30 & -1.59 & - & - & - & - & - \\
\hline TRINITY_DN22965_c1_g3_i1 & $\begin{array}{l}\text { choline } \\
\text { dehydrogenase }\end{array}$ & - & - & - & -2.03 & - & - & - & - \\
\hline TRINITY_DN22965_c1_g6_i1 & $\begin{array}{l}\text { choline } \\
\text { dehydrogenase }\end{array}$ & - & - & - & - & - & 1.52 & 3.74 & 2.16 \\
\hline TRINITY_DN22965_c1_g2_i4 & $\begin{array}{l}\text { choline } \\
\text { dehydrogenase }\end{array}$ & - & - & - & - & - & 0.95 & 2.13 & 1.01 \\
\hline TRINITY_DN22965_c1_g1_i1 & $\begin{array}{l}\text { choline } \\
\text { dehydrogenase }\end{array}$ & - & - & - & - & - & 1.24 & 3.39 & 2.61 \\
\hline TRINITY_DN17620_c0_g1_i1 & $\begin{array}{l}\text { delta 1-pyrroline-5- } \\
\text { carboxylate } \\
\text { synthetase }\end{array}$ & - & - & - & - & 1.27 & 2.69 & 2.01 & - \\
\hline TRINITY_DN33429_c0_g1_i1 & $\begin{array}{l}\text { PAM71 } \\
\text { manganese/calcium } \\
\text { cation transporter }\end{array}$ & - & - & 1.43 & - & - & - & - & - \\
\hline TRINITY_DN14297_c0_g1_i2 & $\begin{array}{l}\text { BASS small solute } \\
\text { transporter }\end{array}$ & 2.24 & 1.16 & 1.70 & 2.02 & - & - & - & - \\
\hline TRINITY_DN22932_c0_g1_i9 & ABC transporter & - & - & 1.34 & - & -4.32 & -4.88 & -3.51 & -1.08 \\
\hline TRINITY_DN21356_c0_g1_i1 & $\begin{array}{l}\text { sodium cation } \\
\text { antiporter }\end{array}$ & 1.29 & 1.81 & 1.56 & 2.06 & - & - & 1.49 & 1.55 \\
\hline TRINITY_DN16027_c0_g1_i1 & $\begin{array}{l}\text { MRS/MGT metal } \\
\text { cation transporter }\end{array}$ & 1.33 & - & - & - & 1.94 & 1.86 & - & - \\
\hline TRINITY_DN15809_c0_g1_i2 & $\begin{array}{l}\text { VCCN chloride anion } \\
\text { channel }\end{array}$ & 1.30 & - & 1.47 & - & -1.91 & - & - & - \\
\hline TRINITY_DN16432_c0_g1_i1 & $\begin{array}{l}\text { MC-type solute } \\
\text { transporter }\end{array}$ & - & - & - & - & 2.67 & 3.42 & - & - \\
\hline TRINITY_DN31363_c0_g1_i1 & $\begin{array}{l}\text { sodium cation } \\
\text { antiporter }\end{array}$ & - & - & - & - & 1.20 & 2.67 & 1.72 & 1.08 \\
\hline TRINITY_DN20714_c0_g1_i3 & $\begin{array}{l}\text { chloride anion } \\
\text { channel }\end{array}$ & - & - & - & - & -3.48 & -2.26 & - & -1.29 \\
\hline TRINITY_DN19909_c0_g1_i1 & $\begin{array}{l}\text { potassium cation } \\
\text { antiporter }\end{array}$ & - & - & - & - & - & 1.81 & - & - \\
\hline
\end{tabular}

Table. $2 \log _{2}$ foldchange value of genes related to general stress responses under different conditions comparing to control 


\begin{tabular}{|c|c|c|c|c|c|c|c|c|c|}
\hline \multirow[t]{2}{*}{ ID } & \multirow[t]{2}{*}{ Annotation } & \multicolumn{4}{|c|}{ Hypo-salinity } & \multicolumn{4}{|c|}{ Recovery } \\
\hline & & $1 \mathrm{~h}$ & $6 \mathrm{~h}$ & $24 \mathrm{~h}$ & $72 \mathrm{~h}$ & $1 \mathrm{~h}$ & $6 \mathrm{~h}$ & $24 \mathrm{~h}$ & $72 \mathrm{~h}$ \\
\hline TRINITY_DN21066_c0_g1_i2 & $\begin{array}{l}\text { M-type thioredoxin } \\
\text { cholroplast }\end{array}$ & - & - & 1.26 & - & - & - & - & - \\
\hline TRINITY_DN21179_c0_g2_i4 & glutathione reductase & 1.69 & 2.13 & 1.27 & 1.80 & - & - & 2.08 & 1.83 \\
\hline TRINITY_DN21179_c0_g2_i1 & glutathione reductase & - & - & - & - & - & 1.74 & - & - \\
\hline TRINITY_DN10934_c0_g1_i1 & Z-type thioredoxin & - & - & - & - & - & 1.32 & - & - \\
\hline TRINITY_DN20657_c0_g1_i2 & ascorbate peroxidase & - & - & - & - & - & 1.57 & - & - \\
\hline TRINITY_DN18599_c0_g1_i2 & Cyclophilin & 1.07 & 1.38 & - & - & - & - & - & - \\
\hline TRINITY_DN21758_c0_g1_i3 & Cyclophilin & 1.38 & - & - & - & - & - & - & - \\
\hline TRINITY_DN19641_c0_g2_i1 & Cyclophilin & 1.06 & 1.14 & - & - & - & - & - & - \\
\hline TRINITY_DN20412_c0_g2_i1 & Cyclophilin & 1.13 & 1.28 & - & - & - & - & - & - \\
\hline TRINITY_DN22159_c0_g1_i1 & Cyclophilin & - & - & 1.98 & - & - & - & - & - \\
\hline TRINITY_DN15044_c0_g1_i2 & Cyclophilin & - & - & - & - & - & 1.39 & - & - \\
\hline TRINITY_DN22176_c1_g1_i3 & HSP70 chaperone & 1.76 & - & - & - & 1.93 & 1.55 & - & - \\
\hline TRINITY_DN22176_c1_g1_i14 & HSP70 chaperone & - & - & - & - & 1.10 & - & - & - \\
\hline TRINITY_DN13473_c0_g1_i1 & $\begin{array}{l}\text { FKBP protein folding } \\
\text { catalyst }\end{array}$ & 1.61 & 1.50 & 1.78 & 1.71 & 1.80 & 1.36 & 1.87 & 2.24 \\
\hline TRINITY_DN22022_c0_g1_i1 & $\begin{array}{l}\text { FKBP protein folding } \\
\text { catalyst }\end{array}$ & 1.29 & - & - & - & 1.27 & - & - & - \\
\hline TRINITY_DN19339_c0_g1_i4 & $\begin{array}{l}\text { FKBP protein folding } \\
\text { catalyst }\end{array}$ & - & - & - & - & 1.02 & 3.11 & 1.86 & - \\
\hline TRINITY_DN22214_c0_g1_i3 & ZRF Hsp40-chaperone & - & - & - & - & - & 1.28 & - & - \\
\hline
\end{tabular}

\section{Figures}

A

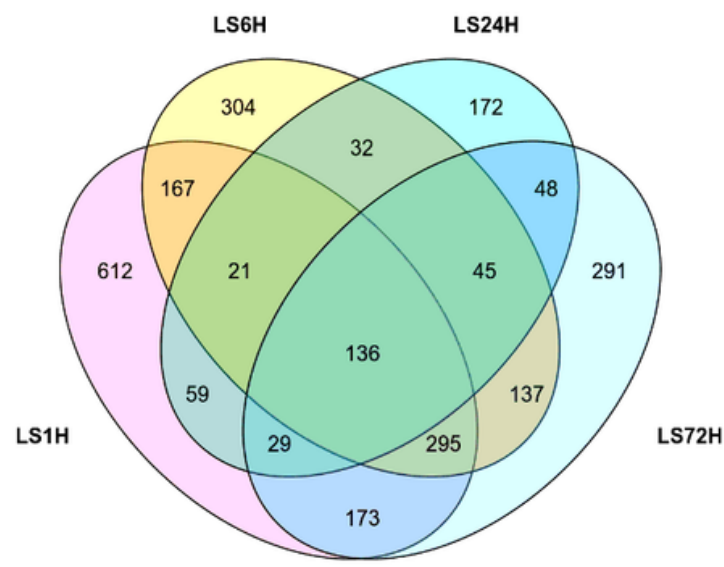

B

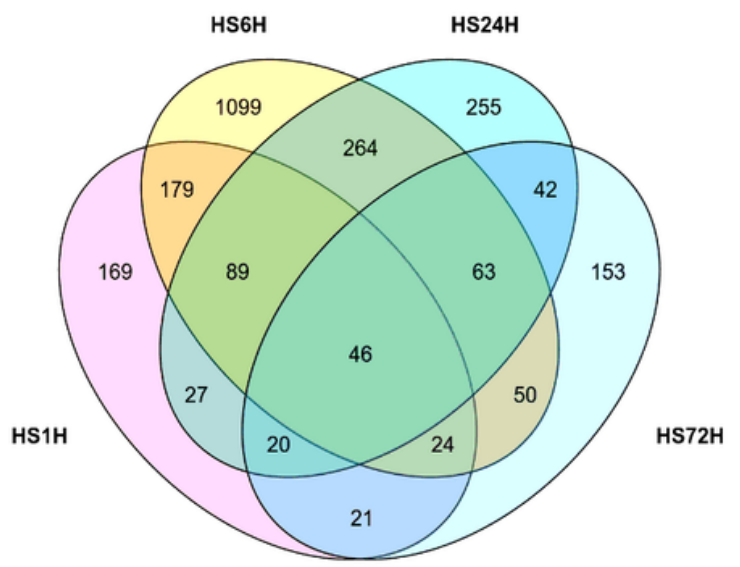

C

\begin{tabular}{lllllllll}
\hline & LS1H & LS6H & LS24H & LS72H & HS1H & HS6H & HS24H & HS72H \\
\hline up & 825 & 587 & 223 & 738 & 263 & 861 & 339 & 239 \\
down & 667 & 550 & 319 & 416 & 312 & 953 & 467 & 180 \\
\hline
\end{tabular}




\section{Figure 1}

Venn diagram of differential expressed genes in different time points of hypo-salinity stress(A) and recovery(B). And summary of DEGs at different time points(C).

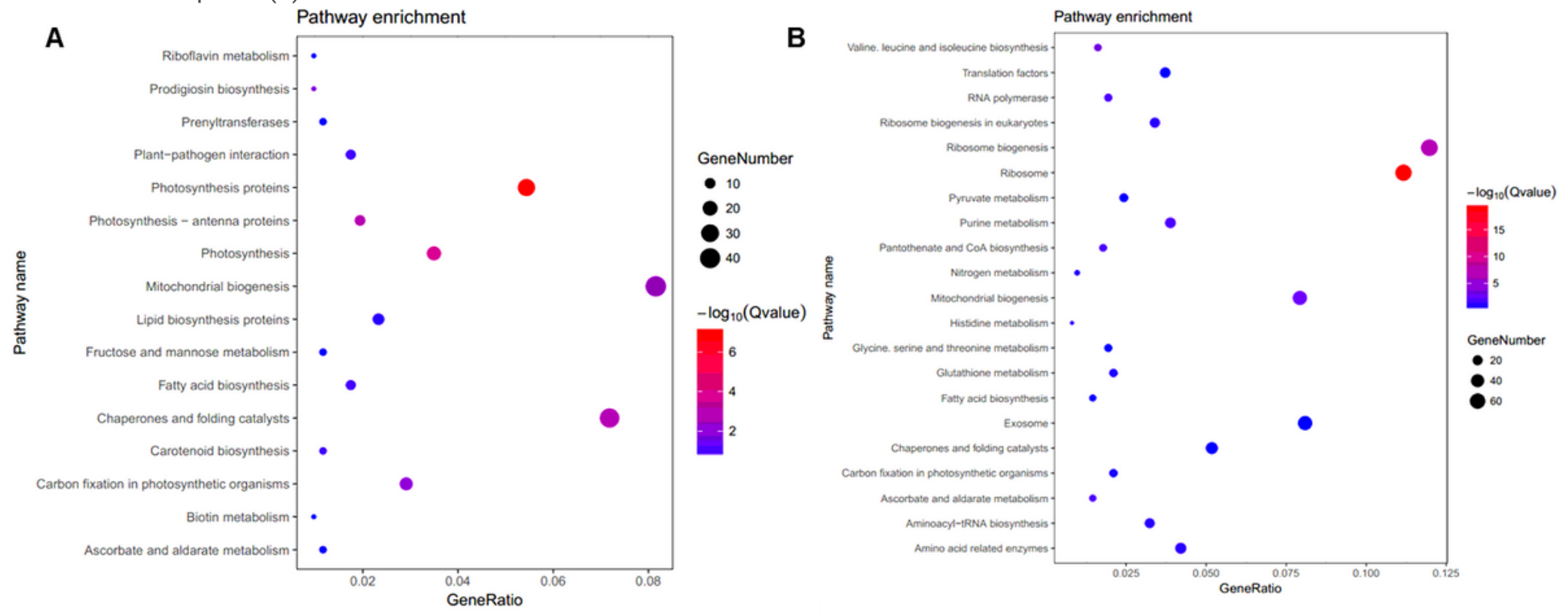

\section{Figure 2}

KEGG enrichment result of DEGs in hypo-salinity stress(A) and recovery(B). 
A

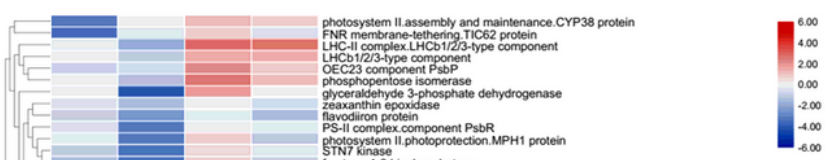

B

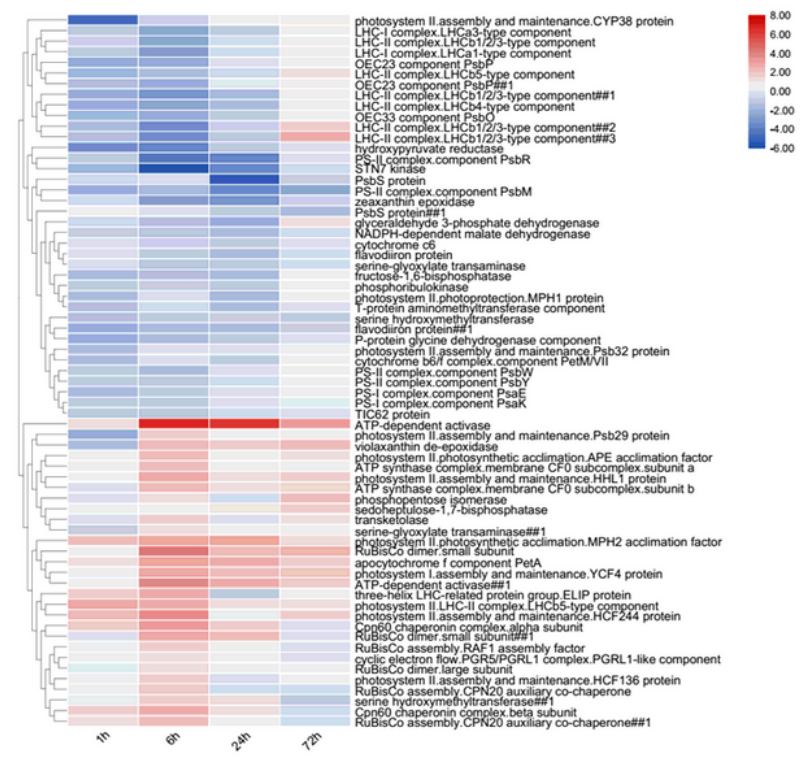

\section{Figure 3}

Heatmap of DEGs involved in photosynthesis pathways under hypo-salinity(A) and recovery(B). 

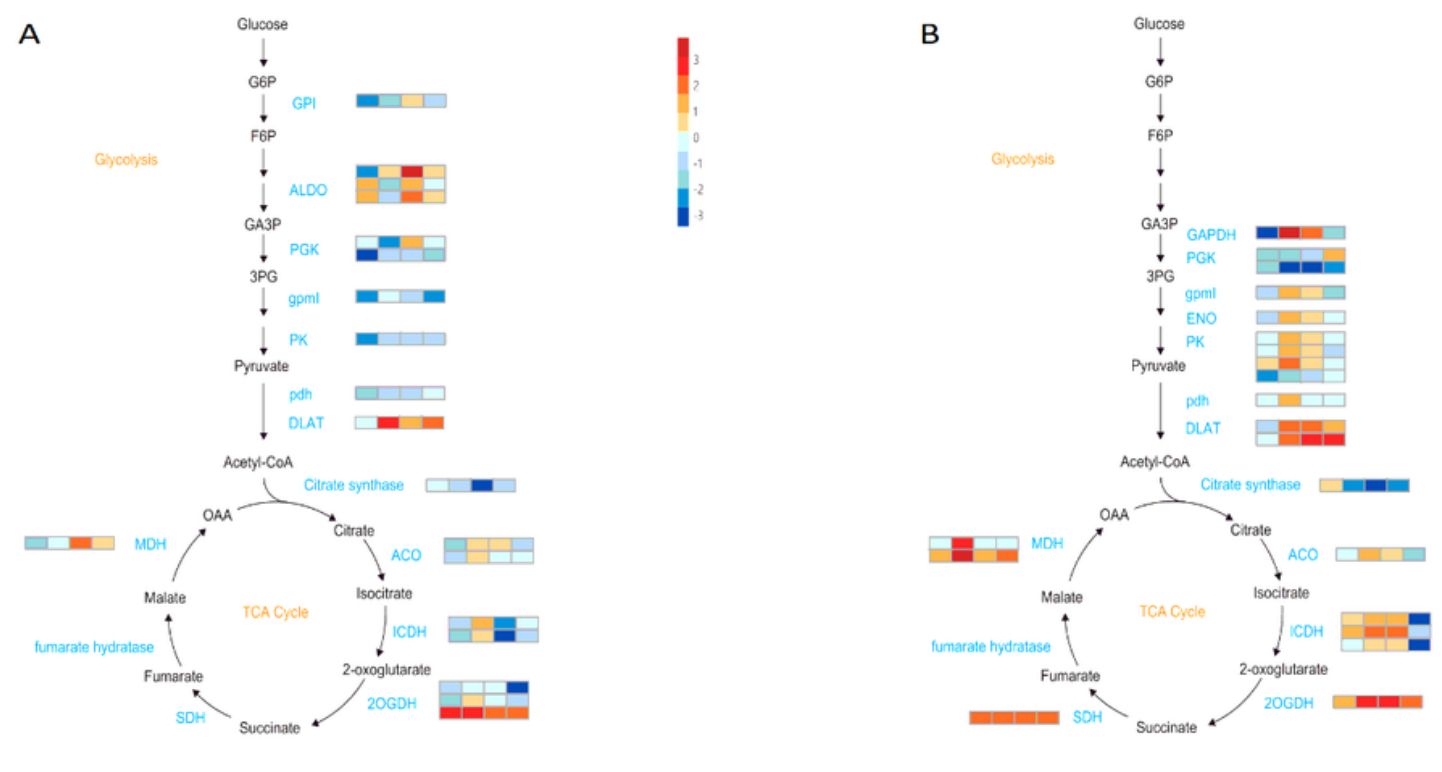

\section{Figure 4}

Expression of DEGs involved in the glycolysis and TCA pathways under hypo-salinity(A) and recovery(B). 


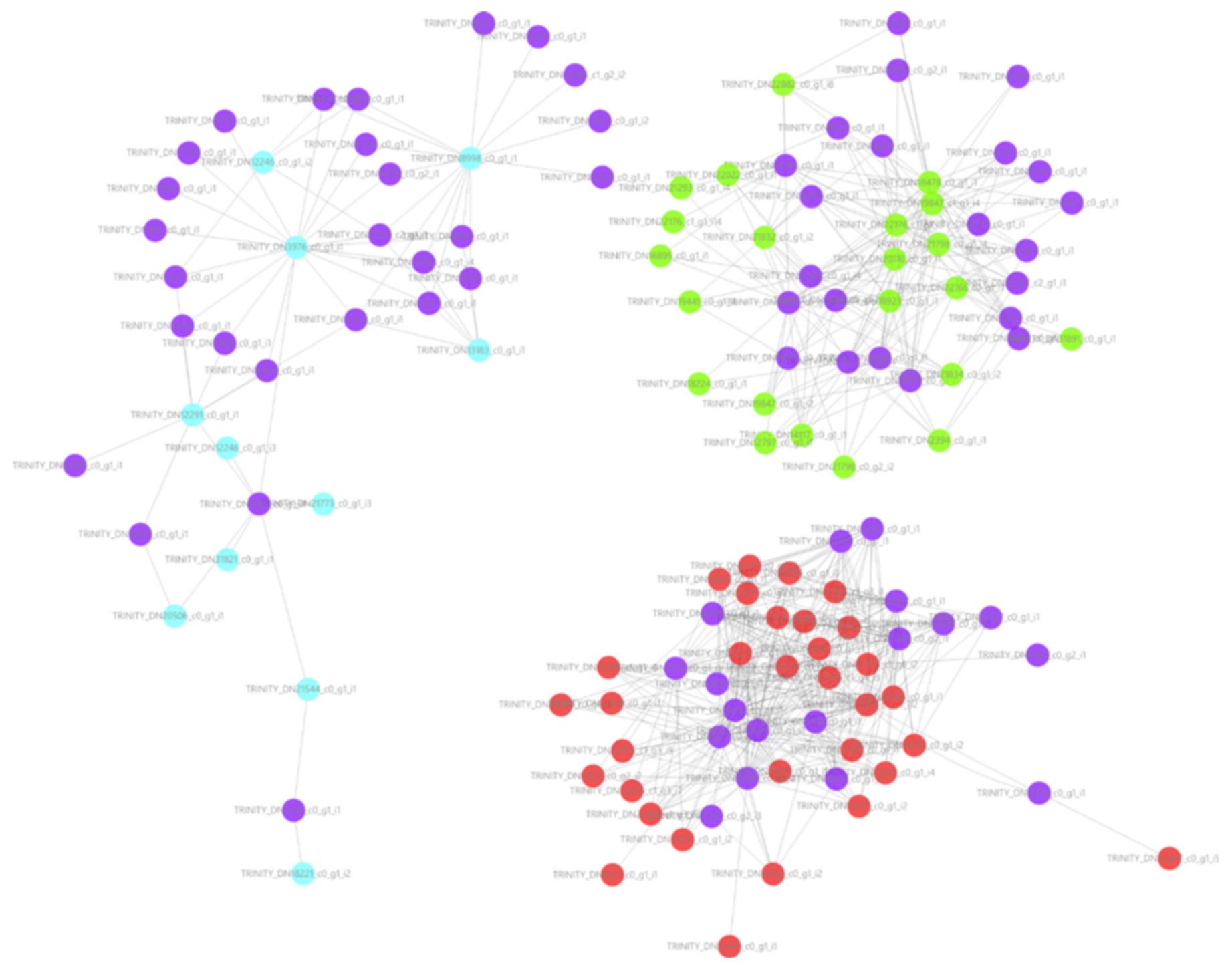

Figure 5

Co-expression network of IncRNA(purple) with mRNA involved in the photosynthesis(cyan), TCA(green) and protein homeostasis(red).

\section{Supplementary Files}

This is a list of supplementary files associated with this preprint. Click to download.

- Additionalfile3.docx

- Additionalfile2.xlsx

- Additionalfile1.xlsx 